\title{
NUMERICAL SIMULATION OF AN EDGE CRACK PROBLEM WITH HOLE OF VARIOUS SHAPES AND SIZES USING MESHLESS METHOD
}

\author{
Ajay Kumar, Sahil Garg, Mohit Pant \\ Department Of Mechanical Engineering, NIT Hamirpur, H.P-177005 \\ Corresponding Author: ajaym9882@gmail.com, \\ Contact No- +91-9882546382
}

\begin{abstract}
This work presents the implementation and extension of element free Galerkin method (EFGM) for modeling and simulation of structures having edge crack with the holes of different shape and sizes and their interaction effects. Intrinsic enrichment criterion is used to model crack tip stress. The nodal data extracted from finite element domain is used to model the EFG domain. The plate is considered to be homogeneous in nature and the size of hole is defined by the ratio of the areas of plate and hole given by $\left(S^{0}=\frac{A^{\text {Halk }}}{A^{\text {Plefs }}}\right)$. Moving least squares interpolants are used to construct the shape function and Lagrange's multiplier is used to enforce the boundary conditions. The hole shapes considered for the simulation are square and the circular and their effect on stress distribution and stress intensity factors (SIFs) is studied. Interaction integral approach is used for numerical evaluation of mode-I SIFs. The results of the simulation provide an insight in avoiding the mode-I crack propagation failure by distribution of stresses via holes.
\end{abstract}

Keywords-Meshless; EFGM; FEM; Stress intensity factors (SIFs).

\section{INTRODUCTION}

In recent years EFG method has been successfully applied to structural and heat conduction problems. EFG method which is applicable to arbitrary shapes, requires only nodal data and no elemental connectivity is needed. In this method moving least squares interpolants are used to construct the trial and test functions for the weak form. Implementation of EFG method increases accuracy also the method does not exhibit any volumetric locking. The convergence rate of EFG method is much higher than the finite element solution

A variety of structural components made of homogeneous or composite materials used now a days in different engineering applications especially in automobile or aerospace industries are generally provided with different shapes of holes for certain operational needs. Due to the stress concentration effect around the hole, cracks are initiated consequently a catastrophic failure may occur. A lot of research concerning these kinds of problems has been done.

Yan X. [1] gave solution of triangular or square hole subjected to internal pressure using boundary element method. Yan X.[2]used displacement discontinuity method for numerical analysis of cracks emanating from a circular or square hole under tension. Elvin B. Shields [3] developed the new techniques for solving fracture problems involving multiple cracks using different computer models by FEM. Hasebe N. and Ueda N[4] used FEM solution for calculating the SIFs of the crack problem with oriented square holes. Yan X. [5] gave the solution for a crack emanating from a corner of a square hole in an infinite plate using the hybrid displacement discontinuity method. Yan X. [6]used numerical method FEM calculating the SIFs of a branched crack. Yan X. [7] used FEM techniques to give solution for a problem of crack emanating from a square hole in a rectangular plate under biaxial loads. Yan X. [8] used a numerical approach to solve the multiple crack problem and calculate the effect on SIFs due to multiple cracks interaction. Tweed and Rooke[9] used the mellin transform technique to make analysis of bi-axial tensions for a branching crack emanating a circle hole.Evans R. et al. [10]developed new geometry correction factors using a p-version finite element method program.

In this paper, detail solution of SIFs using EFG method and FEM technique of edge crack problem with circular and square holes elastic crack problems are given, which can reveal the effect of geometric parameters of the cracked bodies on the SIFs.

\section{REVIEW OF EFGM}

In EFGM, a field variable $\mathrm{u}$ is approximated by moving least squares(MLS) approximation function $u^{\text {h }}(x)$ [11], which is given by:

$$
u^{h}(\mathbf{x})=\sum_{j=1}^{m} p_{j}(\mathbf{x}) a_{j}(\mathbf{x}) \equiv \mathbf{p}^{T}(\mathbf{x}) \mathbf{a}(\mathbf{x})
$$

Where, $\mathbf{p}(\mathbf{x})$ is a vector of basis functions, $\mathbf{a}(\mathbf{x})$ is unknown coefficients, and $m$ is the number of terms in the basis. The unknown coefficients $\mathbf{a}(\mathbf{x})$ are obtained by minimizing a weighted least square sum of the difference between local approximations, $u^{\text {h }}(x)$ and field function nodal parameter $u_{I}$ The weighted least square sum denoted by $L(\mathbf{x})$ can be written in following quadratic form: 


$$
L(\mathbf{x})=\sum_{I=1}^{n} w\left(\mathbf{x}-\mathbf{x}_{I}\right)\left[\mathbf{p}^{T}(\mathbf{x}) \mathbf{a}(\mathbf{x})-u_{I}\right]^{2}
$$

where, $u_{I}$ is the nodal parameter associated with node $I$ at $\mathbf{x}=\mathbf{x}_{I}$ but these are not the nodal values of $u^{h}\left(\mathbf{x}=\mathbf{x}_{I}\right)$ because $u^{h}(\mathbf{x})$ as an approximant not an interpolants (the difference between $u_{I}$ and $u^{h}\left(\mathbf{x}=\mathbf{x}_{I}\right), w\left(\mathbf{x}-\mathbf{x}_{I}\right)$ is the weight function having compact support associated with node $I$, and $n$ is the number of nodes with domain of influence containing the point $\mathbf{x}$, i.e. $w\left(\mathbf{x}-\mathbf{x}_{I}\right) \neq 0$ By setting $\frac{\partial L}{\partial \mathbf{a}}=0$, following set of linear equation is obtained

\section{$\mathbf{A}(\mathbf{x}) \mathbf{a}(\mathbf{x})=\mathbf{B}(\mathbf{x}) \mathbf{u}(3)$}

By substituting Eq. (3) in Eq. (1), the approximation function is obtained as:

$$
u^{h}(x)=\sum_{I}^{n} \phi_{I}(x) u_{I}(4)
$$

The essential boundary conditions are imposed using Lagrange's multiplier method[12].

\section{STRESS INTENSITY FACTOR COMPUTATION}

The numerical simulation of stress intensity factor has been done by J-Integral approach.Rice [13] proposed a path independent energy-momentum tensor that could be used as a fracture parameter when the contour enclosed a crack tip. The $J$ integral is given by:

$J=\int_{\Gamma}\left[W \delta_{1 j}-\sigma_{i j} \frac{\partial u_{i}}{\partial x_{1}}\right] n_{j} d \Gamma$

By using green's theorem the domain form of the $J$ integral is given as:

$J=\int_{A}\left[\sigma_{i j} \frac{d u_{i}}{d x_{1}}-W \delta_{1 j}\right] \frac{\partial q}{\partial x_{j}} d A$
The $J$ integral is equal to the energy release rate for elastic materials. For a general mixed-mode case $J$ integral is defined as:

$J=G=\frac{1}{E^{\prime}}\left(K_{I}^{2}+K_{I I}^{2}\right)(7)$

By further expansion the mixed mode intensity factor has been calculated by

$M^{(1,2)}=\frac{2}{E^{\prime}}\left(K_{I}^{(1)} K_{I}^{(2)}+K_{I I}^{(1)} K_{I I}^{(2)}\right)(8)$

Computing $M^{(1,2)}$ from Eq. (8) with some numerical adjustments for auxiliary fields allows the solution for the mode 1 stress intensity factor as

$K_{I}^{(1)}=\frac{2}{E^{\prime}} M^{(1,2)}$

\section{RESULTS AND DISCUSSION}

In this work plate of length, $\mathrm{L}=2$ units and width, $\mathrm{W}=3$ units with an edge crack (crack length $\mathrm{a}=0.3$ ) is considered. The Young modulus of elasticity, $\mathrm{E}$ and poission ratio, $v$ of plate is considered to

be $210 \times 10^{5}$ and 0.3 respectively. The shape of hole is considered to be circular and square respectively. The hole is placed at the center of the plate. The area of hole is considered in the ratio of $\frac{1}{10}, \frac{1}{15}, \frac{1}{20}$ respectively with respect to the area of the plate. The simulations are performed for about 1400 nodes and 6 point Gauss quadrature is used for numerical integration of domain. The geometry of the problem is shown in Fig.1. The tensile load $\sigma$ considered for analysis is 50000 units applied at top edge of the plate while bottom edge of plate is fixed.

\section{A. Analysis of Edge Crack with Circular hole}

The results yielded after the numerical simulation of edge crack problem with circular hole of varying sizes show an ascending trend with reduction in size of the hole. The deformed shape generated by EFG simulation for this configuration is represented in Fig.2. The calculated values of stress intensity factors (SIFs) using EFGM represented in Table.1. The values of SIFs using EFG method shows good agreement with the values obtained by the FEM as shown in Fig.4. 


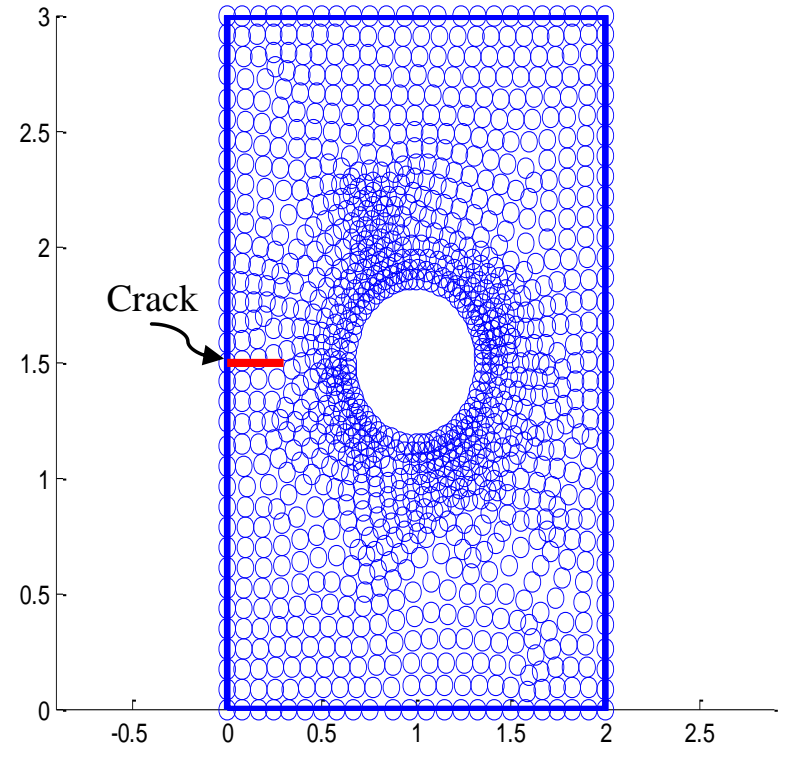

(a)

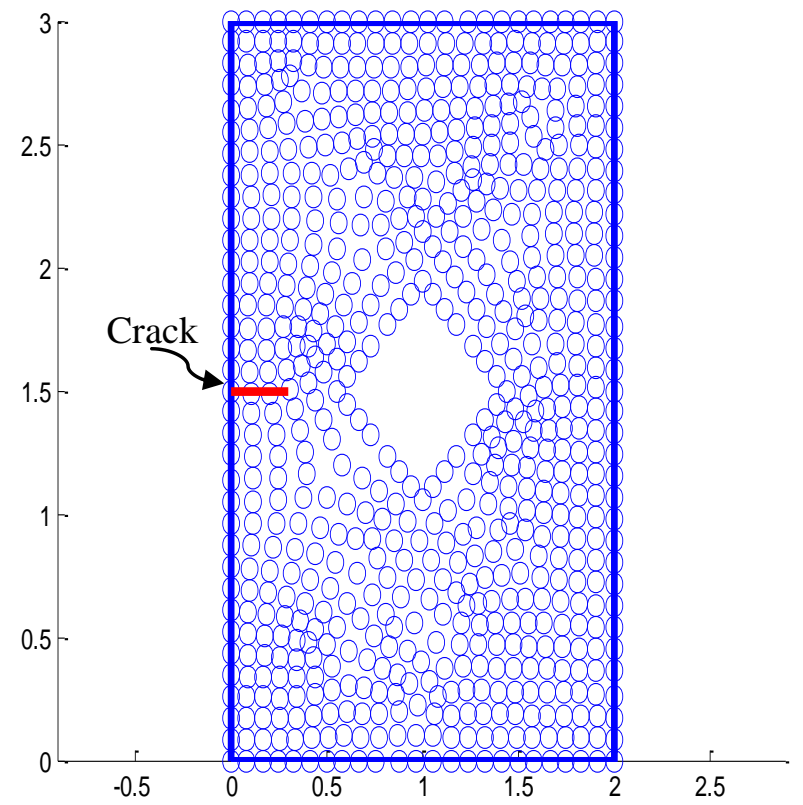

(b)
Table 1. Values of SIF though EFGM for circular hole

\begin{tabular}{|l|l|l|l|l|}
\hline $\begin{array}{l}\text { S. } \\
\text { No. }\end{array}$ & $\left(\frac{A^{\text {Hole }}}{A^{\text {Plate }}}\right)$ & $\begin{array}{l}\text { Radius of } \\
\text { circular } \\
\text { hole }\end{array}$ & $\begin{array}{c}\text { SIF } \\
(\mathrm{KI})\end{array}$ & $\begin{array}{l}\text { SIF } \\
(\mathrm{KII})\end{array}$ \\
\hline 1. & $1 / 20$ & 0.3090 & 1.159 & $\times 80382$ \\
\hline 2. & $1 / 15$ & 0.3568 & 1.240 & $\times 600677$ \\
\hline 3. & $1 / 10$ & 0.4370 & 1.473 & 2200840 \\
\hline
\end{tabular}

\section{B. Analysis of Edge Crack with Square hole}

The results yielded after the numerical simulation of edge crack problem with square hole of varying sizes show an ascending trend with reduction in size of the hole. The square is oriented by an angle of $90^{\circ}$. The deformed shape generated by EFG simulation for this configuration is represented in Fig.3. The calculated values of stress intensity factors (SIFs) using element free Galerkin (EFG) method represented by the Table.2. The values of SIFs using EFG method shows good agreement with the values obtained by the FEM as shown in Fig.5.

Table 2. Values of SIF though EFGM for square hole

\begin{tabular}{|l|l|l|l|l|}
\hline $\begin{array}{l}\mathrm{S} . \\
\mathrm{N} \\
\text { o. }\end{array}$ & $\left(\frac{A^{\text {Hole }}}{A^{\text {Plate }}}\right)$ & $\begin{array}{l}\text { Side } \\
\text { of } \\
\text { square } \\
\text { hole }\end{array}$ & $\begin{array}{l}\text { SIFs } \\
(\mathrm{KI})\end{array}$ & $\begin{array}{l}\text { SIFs } \\
(\mathrm{KII})\end{array}$ \\
\hline 1. & $1 / 20$ & .5475 & $1.2319 \times 1027.02$ \\
\hline 2. & $1 / 15$ & .6324 & $1.3339 \times 11041.68$ \\
\hline 3. & $1 / 10$ & .7744 & $1.7005 \times 129.002$ \\
\hline
\end{tabular}

Fig.1. Problem Geometry (a) Circular hole (b) Square hole. 


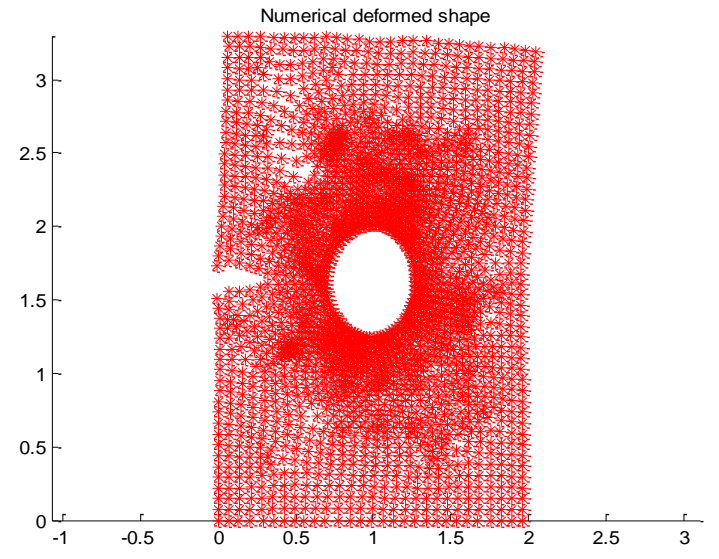

$\mathrm{Fi}$

g.2 Deformed shape of plate with edge crack and circular hole.

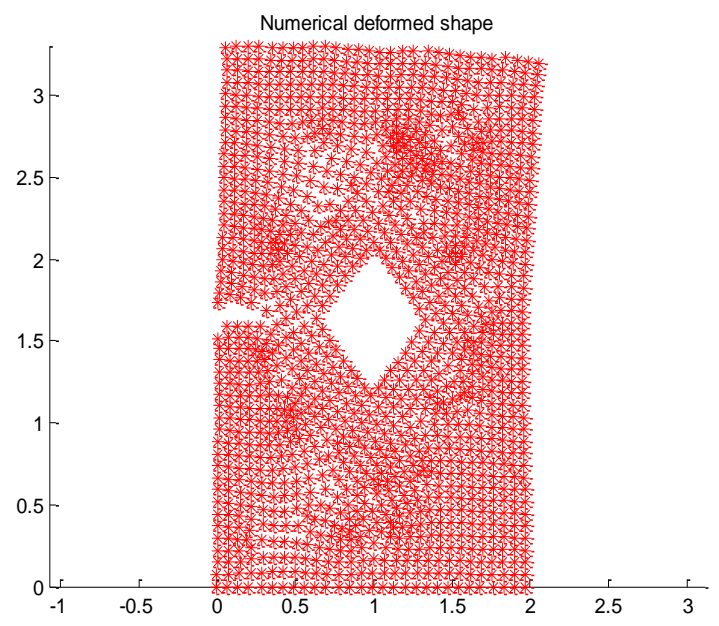

Fig.3Deformed shape of plate with edge crack and square hole

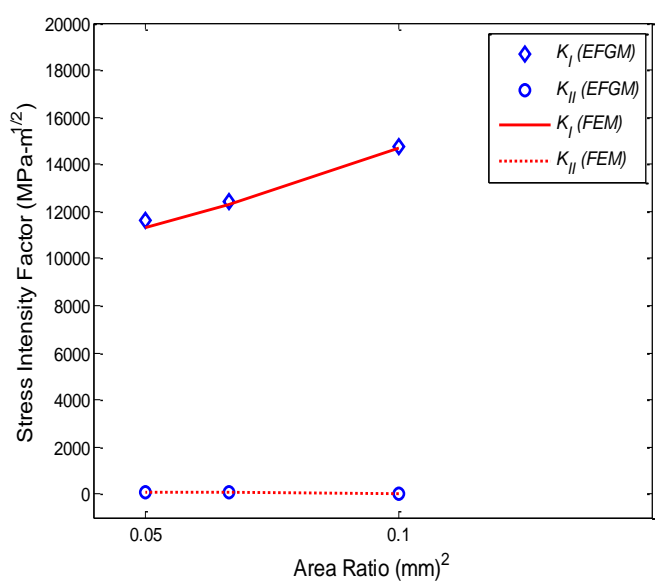

Fig.4 Comparison of SIFs through EFGM and FEM for circular hole.

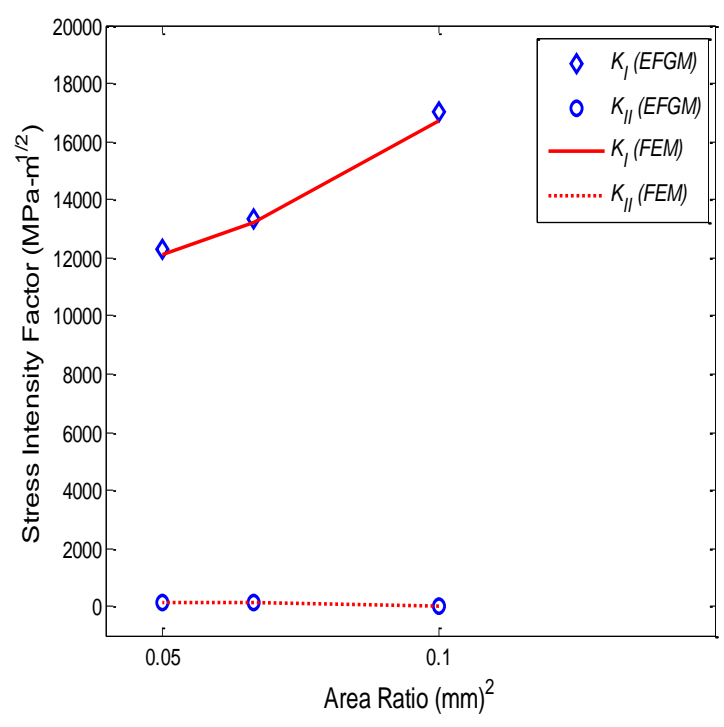

Fig.5 Comparison of SIFs through EFGM and FEM for square hole.

\section{CONCLUSION}

The simulation results show that holes with circular shapes have reduced SIFs due to symmetrical geometry thereby avoiding the failure of material in comparison to square holes. The simulations help us understand the variation of SIFs for various geometries with holes and also establish the prowess of EFG method in modeling strong discontinuities in one domain. This method can be further used to model and simulate more complex geometries in non-homogeneous domains.

\section{References}

[1] X. Yan, "Stress intensity factors for cracks emanating from a triangular or square hole in an infinite plate by boundary elements," vol. 12, pp. 362-375, 2005.

[2] X. Yan, "Cracks emanating from circular hole or square hole in rectangular plate in tension," vol. 73, pp. 1743-1754, 2006.

[3] E. B. Shields, "Fracture prediction of hole patterns with multiple cracks using the finite element method," vol. 23, pp. 13-20, 2001.

[4] N. Hasebe and M. Ueda, "Crack originating from a corner of a square hole," Eng. Fract. Mech., vol. 13, no. 4, pp. 913-923, 1980.

[5] X. Yan, "Analysis for a crack emanating from a corner of a square hole in an infinite plate using the hybrid displacement discontinuity method," vol. 28, pp. 835-847, 2004.

[6] X. Yan, "An Efficient and Accurate Numerical Method of Stress Intensity Factors Calculation of a," vol. 72, no. May 2005, 2016.

[7] X. Yan, "A numerical analysis of cracks emanating from a square 
hole in a rectangular plate under biaxial loads," vol. 71, pp. 16151623,2004

[8] X. Yan, "An Effective Numerical Approach for Multiple VoidCrack," vol. 73, no. July 2006, pp. 525-535, 2016.

[9] J. Tweed and D. P. Rooke, "The distribution of stress near the tip of a radial crack at the edge of a circular hole," Int. J. Eng. Sci., vol. 11, no. 11, pp. 1185-1195, 1973.

[10] R. Evans, A. Clarke, R. Gravina, M. Heller, and R. Stewart, "Improved stress intensity factors for selected configurations in cracked plates," Eng. Fract. Mech., vol. 127, pp. 296-312, 2014.

[11] Y. Y. Lu, T. Belytschko, and L. Gu, "A new implementation of the element free Galerkin method," Comput. Methods Appl. Mech. Eng., vol. 113, no. 2, pp. 397-414, 1994.

[12] V. P. Nguyen, T. Rabczuk, S. Bordas, and M. Duflot, "Meshless methods: A review and computer implementation aspects," Math. Comput. Simul., vol. 79, no. 3, pp. 763-813, 2008.

[13] J. R. Rice, "A Path Independent Integral and the Approximate Analysis of Strain Concentration by Notches and Cracks," J. Appl. Mech., vol. 35, no. 2, p. 379, 1968. 